\title{
dialog-gesundheit - le pouvoir de la question pour une nouvelle compétence santé
}

Michael Deppeler

Correspondance:

Dr Michael Deppeler SaluToMed.AG

Kirchlindachstrasse 7

CH-3052 Zollikofen

Tél. 0319112060

Fax 0319116312

m.deppeler@hin.ch
Warum syt dir so truurig? Förchtet der das, wo no chönnt cho?

Aber dir syt doch versicheret gäge die mügleche Zuefäll Und wenn ds Alter de chunnt, heit dir de ä rächti Pension

Warum syt dir so truurig? Nei dir wüsset ke Grund

Vilicht, wenn der e Grund hättet, wäret der weniger truurig

Mänge, wenn ds Läben ihm wehtuet, bsinnt sech derdür wider dra

(Mani Matter)

En décembre 2009, nous avons fêté les 50 ans du rock bernois au Berner Bierhübeli. Pour clore cette célébration, Polo Hofer a interprété la dernière chanson de Mani Matter. Le père fondateur du rock en dialecte, toujours provocateur, a ainsi rendu un nouvel hommage au troubadour trop tôt disparu. Mais quel rapport ces deux musiciens et acrobates des mots ont-ils avec dialog-gesundheit et la médecine? Ils narrent des histoires et posent des questions qui incitent à la réflexion.

Pourquoi êtes-vous si tristes? Nous médecins de famille, sommes-nous également tristes? Ou simplement en colère? Et pour quelle raison finalement? Avonsnous des problèmes et soucis différents des autres? Différents de ceux de nos patients?

Aurons-nous encore besoin de médecins de famille dans 10 ans? C'est à cette dernière question provocatrice que 5 médecins de famille ont été confrontés en 2003 lors du $20^{\mathrm{e}}$ anniversaire de l'Instance bernoise pour la médecine de famille. De quels médecins de famille aurons-nous besoin dans 10 ans? De quelle médecine de premier recours et de quelle médecine en général? Nous avons tenté d'apporter de premières réponses à ces questions dans le cadre de deux ateliers animés auxquels ont également participé des confrères ayant affaire à la médecine de famille: de plus en plus complexe, le système de santé devient difficile à contrôler, du moins «par le haut». Ce secteur est l'un des principaux employeurs de Suisse; axé sur le profit, il n'affiche aucune véritable volonté d'économie. Mais que fait-on des patients et de leurs souffrances? Lors de l'atelier, le patient qui devrait être au centre de nos préoccupations nous est régulièrement «sorti de l'esprit». Ce constat douloureux, mais finalement précieux du «patient oublié» a été le point de départ de swissdoc qui est progressivement devenu dialog-gesundheit au cours des six dernières années. (www.dialog-gesund heit.ch). Depuis, des médecins et la population se réunissent périodiquement pour dialoguer autour de questions liées à la santé posées par des patients et leur environnement, les familles, les quartiers ou les communes. La première rencontre a eu lieu en 2004 dans le forum dialog-gesundheit de Zollikofen (anciennement swissdoc.forum). Depuis 2006, les rencontres ont lieu dans le forum dialog-gesundheit de Frutigland (avec une pause en 2009). Un forum de 90 minutes est organisé, animé et documenté tous les deux mois. A la fin de chaque forum, on tente de définir ensemble les questions et le thème de la prochaine édition. Ces forums prennent ainsi la forme de «cercles de qualité sur le système de santé» ouverts à la population, sans le risque d'une trop grande appropriation du thème par sa direction.

L'exposé du Professeur Syme (Université de Berkeley) lors de la $7^{\mathrm{e}}$ Conférence suisse sur la promotion de la santé en 2005 à Fribourg a été un événement clé. Il raconta son histoire personnelle consacrée à la promotion de la santé et de la prévention en Californie, un parcours semé d'échecs en quête de nouvelles voies. Lui aussi s'est intéressé au poids de la maladie («burden of disease») répandu dans toute la population, a lancé d'innombrables campagnes de prévention pour être sans cesse déçu par un succès médiocre. Après avoir analysé ces échecs, il décida enfin de songer à quelque chose de nouveau: il interrogea la population sur ses besoins en matière de santé. Elle lui livra la réponse suivante - peu surprenante pour nous, les médecins de famille: «nos problèmes sont complètement différents de ce que vous, universitaires, pensez. (Il en va de même en politique.) Nous avons des soucis avec l'éducation de nos enfants et notre relation sentimentale, nous sommes confrontés à des difficultés financières et des conflits irrésolus sur notre lieu de travail, du moins pour ceux d'entre nous qui ont encore un travail. Nous avons d'abord besoin de soutien pour résoudre ces problèmes quotidiens; ce n'est qu'ensuite que nous serons disposés à arrêter de fumer, de boire ou de manger. Nous savons que cela est nocif pour notre santé.»

C'est ainsi que la Californie reçut son premier «Wellness guide». Mais l'évaluation donna un résultat décourageant. Ce guide n'eut pas plus d'impact que les autres programmes de prévention. Le Professeur Syme reprit son courage à deux mains et réinterrogea la population: que pouvons-nous faire mieux? Quelle est notre mission? Il s'avéra que la population avait été complètement dépassée par le premier guide trop difficile d'accès (trop de texte, trop compliqué). Elle souhaitait des textes courts, des photographies et des listes 
de contrôle claires. La seconde édition a été un succès, notamment auprès de la population la plus défavorisée. Les personnes des couches sociales plus élevées recherchent de toute façon des solutions par ellesmêmes, comme nous le constatons dans les consultations.

Parallèlement, un événement remarquable s'est produit en 2005 lors du forum de Zollikofen, lorsque les participants ont exprimé leurs trois principales revendications en matière de santé. A savoir:

- la coordination des offres de la commune et de la région en matière de promotion de la santé. La première conférence dialog-gesundheit 2006 a répondu à cette revendication;

- un interlocuteur au niveau de la commune susceptible de donner les renseignements de base sur les offres de santé. Peu de temps après, un poste à $20 \%$ a été créé parmi le pool d'emplois du Département des affaires sociales, auquel est rattachée la santé; de la santé qui font aujourd'hui l'objet de nombreuses discussions. Ils reflètent de façon saisissante ce qui a été étudié et reconnu ces dernières années sur le plan théorique: la santé et les maladies ne doivent plus être réduites à un simple schéma monocausal. Il s'agit toujours de processus biopsychosociaux complexes et le plan systémique doit impérativement être pris en compte. C'est le quotidien et c'est la vie. Une autre dimension, souvent ignorée, fait également partie de la vie: les valeurs qui s'expriment souvent aussi par une quête spirituelle (pas uniquement religieuse). Toutes ces questions et dimensions trouvent progressivement leur place dans l'intimité d'un forum. En 2009, la discussion a porté, par exemple, sur la question suivante: Quel est le lien entre la réconciliation et la santé/ maladie?

dialog-gesundheit souhaite une promotion de la santé et de la prévention directement à la base en réponse aux questions et soucis qui préoccupent véritablement la population. Nous complétons ainsi toutes

\section{On ne peut jamais résoudre un problème avec le même type de pensée qui a créé le problème (Albert Einstein)}

- Une meilleure information sur les questions liées à la santé. L'ancien guide pratique alémanique «Chummerzhilf» avec ses rares adresses de services d'urgence ne répondait plus depuis longtemps aux besoins actuels. Nous collaborons depuis avec dialog-gesundheit à l'élaboration du «Guide pratique pour la santé et la maladie», un projet soutenu par la Promotion suisse de la santé en tant que projet innovant.

Ces différentes mesures, très significatives et surtout proches de la base, qui ont été prises dans le cadre d'un dialogue dirigé et documenté, ont permis à des projets de santé individuels, mais aussi collectifs de voir le jour. Ces projets s'inspirent des facteurs déterminants

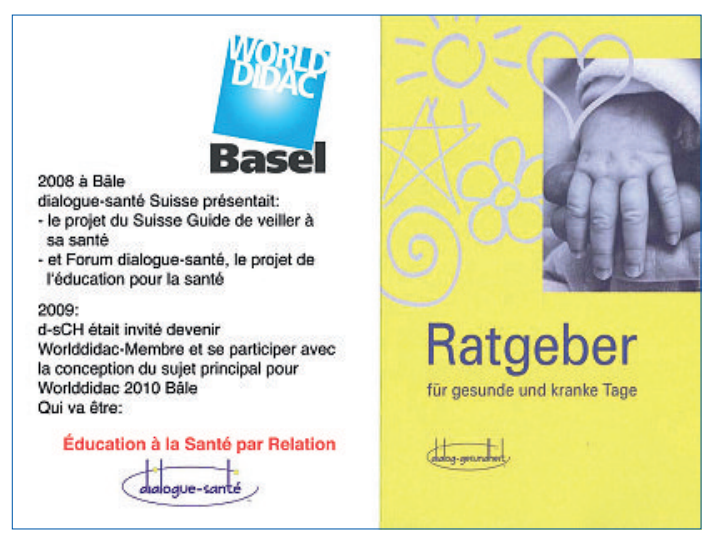

Le guide pratique sur les principales questions liées à la santé a vu le jour à la demande de la population. les «solutions top-down» qui visent en premier lieu la prévention centrée sur le contexte de vie (par ex. interdiction de fumer, interdiction de publicité, etc.), mais malheureusement très peu la prévention centrée sur le comportement; ici, une nouvelle voie, une nouvelle réflexion s'impose. Dans un processus de longue durée, le patient et la population sont invités et motivés à l'autoresponsabilité: dans le forum, dans le dialogue, avec le temps aussi dans d'autres domaines de la santé publique et enfin, «tout naturellement» en consultation. Nous avons tous ainsi la possibilité - et la liberté - de découvrir de nouvelles solutions et ressources dans un monde complexe qui nous est de plus en plus imposé par de nouvelles lois, ordonnances et directives. Des aspects urgents de santé publique et de politique sanitaire et sociale s'en trouveront ainsi renforcés, notamment pour contrebalancer les stratégies orientées vers le profit et l'économie de marché d'autres acteurs. C'est pour cette raison que dialog-gesundheit tient absolument à rester indépendant. D'où le développement d'une propre plateforme de savoir qui permettra d'évaluer l'ensemble du processus, mais aussi des aspects partiels. En fin de compte, dialog-gesundheit est un énorme processus systémique de communication et de solution qui, selon nous, deviendra finalement adéquat, efficace et, qui sait, même économique. Rien que pour la simple raison que nous avons tous travaillé selon le principe du bénévolat; mais en cela sera-t-il toujours ainsi? Ce travail nous paraît-il suffisamment précieux? Et la question se pose à tous les participants. 
Le souhait de la population envers un lien renforcé entre santé et formation est un constat essentiel des années 2008 et 2009. Ce lien est souhaité dès l'école, puis tout au long de la vie grâce à une formation pour adultes, notamment dans le secteur quaternaire comme par exemple dialog-gesundheit et ses forums. La formation réclame, elle aussi, de nouvelles voies, similaires de façon surprenante (?) aux nouveaux jalons posés dans le système de santé: abandon de la «relation patriarcale enseignant-élève» au profit d'un développement commun de nouvelles compétences. Par le biais de la recherche sur la résilience, on connaît la notion de pédagogie dérivée du concept de l'apprentissage lié à l'expérience, sustained shared thinking: il s'agit d'un échange mutuel et durable entre l'éducateur et l'enfant afin de déclencher un processus de réflexion qui s'appuie sur des questions encore ouvertes (!) pour inciter à la réflexion, à la recherche de solution aux problèmes ou au dialogue. Apparemment, $95 \%$ des questions posées dans les écoles sont des questions fermées. La situation est-elle vraiment beaucoup mieux dans le système de santé? Cet échange mutuel incite à la prise de conscience des intérêts et des perspectives propres et d'autrui, en instaurant un dialogue d'égal à égal, une écoute active et une attention nouvelle en vue de jeter de véritables ponts entre les deux protagonistes. Chaque acteur se considère comme un apprenant et fait l'expérience de cet apprentissage. L'autre est le spécialiste de sa vie et de son histoire. Ensemble, nous pouvons apprendre beaucoup l'un de l'autre.

La valeur de la «narration» (médecine narrative) a été reconnue depuis longtemps sur les plans les plus divers, même dans la médecine de famille. Aucun matériel particulier, comme une présentation PowerPoint ou un vidéoprojecteur, n'est nécessaire. Dans le forum, nous sommes assis en cercle et seul un tableau à feuilles mobiles sert à noter les points importants (notamment aussi en vue du procès-verbal). Le pouvoir de la narration et de l'écoute suffit. L'exercice répété devant un auditoire permet aux participants d'acquérir également la capacité à mieux exprimer leurs soucis et leurs craintes, mais aussi leurs besoins et leur histoire. La narration s'effectue à deux niveaux: une participante raconte son histoire et la manière dont elle résout sa tâche de vie. L'animateur narre des histoires qui comportent, entre autres, des solutions possibles. La relation entre les personnes et au sein du groupe constitue la propre histoire de ce groupe. Le partage et l'extériorisation verbale de la souffrance provoquent d'autres narrations qui élargissent la liberté d'action des participants et du groupe, contribuant

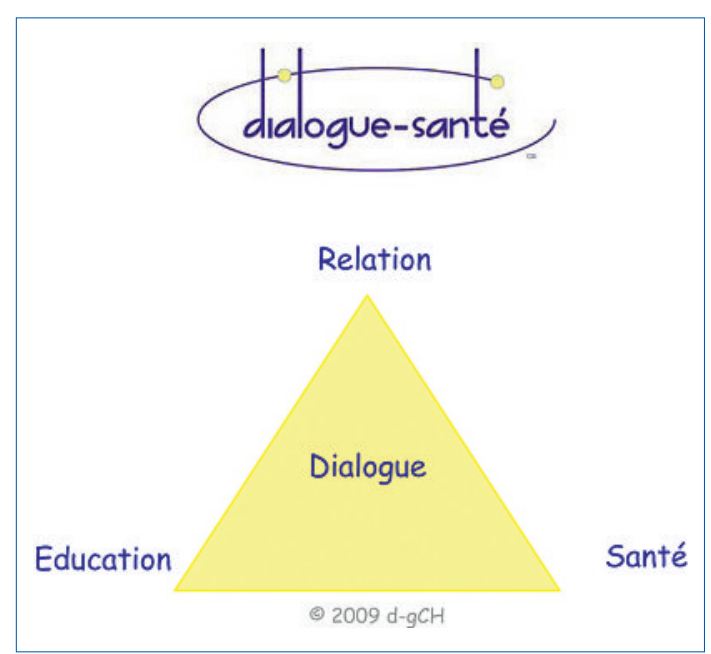

d'une certaine manière à résoudre leurs problèmes et à vivre finalement plus sainement.

Ainsi, dialog-gesundheit relève toujours d'une approche saluto-génétique. Cette approche peut justement s'avérer déterminante lorsqu'il s'agit de porter la promotion de la santé et de la prévention sur le devant de la scène politique ou auprès de la population. Ce processus a lieu à trois niveaux:

- la compréhension: la compréhension doit être garantie. Elle dépend de la langue et de la culture et implique des possibilités d'accès, de l'école jusqu'à la vieillesse. L'information et la communication sont essentielles;

- l'action: où sont les ressources, à savoir renforcer les stratégies de «coping» et de l'autogestion, soutenir le processus individuel de développement et de maturité, mais aussi participer à des «ressources externes» dont la coordination optimisée des «assistants» (soins intégrés);

- le sens: qu'est-ce qui me fait du bien? Qu'est-ce qui ne me fait pas de bien? Comment puis-je trouver un sens pour moi et mon environnement, quelle est ma raison de vivre, pour quelle raison est-ce que je souhaite rester en bonne santé ou le devenir?

Warum syt dir so truurig? Wohl, me gseht nech's doch a. Söttet emal öiji Gsichter gseh, wenn dr sitzet im Büro. Söttet emal öiji Gsichter gseh, wenn der fahret im Tram. Warum syt dir so truurig? S'geit doch so wi der's weit. Frou u Chind sy doch zwäg, im Pruef geit's geng e chly vorwärts,

s'längt doch ou hie und da scho für nes chlys Drübery. (Mani Matter) 